\title{
Study on a Determination of Measures Needed to Improve the Quality of Analyzes Performed in a Chemical Laboratory
}

\author{
LILIANA LUCA ${ }^{1 *}$, MINODORA MARIA PASARE ${ }^{1}$, RUXANDRA ELENA LUCA ${ }^{2}$, AMALIA VENERA TODORUT ${ }^{1}$ \\ 'University Constantin Brancusi of Targu-J iu, 1 Republicii Str., Targu-jiu, Romania \\ 2University of Medicine and Pharmacy Victor Babes Timisoara, 2 Eftimie Murgu Sq., 300041, Timisoara, Romania
}

\begin{abstract}
In this paper the authors present a study on the use of the tree diagram to solve a quality problem in a chemical laboratory. The study aims to identify actions/measures that ensure elimination of sources of errors that cause non-quality problems in analyzes performed in a chemical laboratory. Several measures have been identified that have been grouped into four categories corresponding to four specific objectives. There was a relatively complex 4-level tree diagram. The specified tree chart is useful for organization management and staff in the chemical laboratory to prevent and eliminate nonconformities and generally to improve the quality of the deployed activities.
\end{abstract}

Keywords: chemical laboratory, analyzes, quality, tree diagram

In many areas of activity, chemical analysis plays a particularly important role. Chemical analysis determines the composition of substances, controls the quality of raw materials needed in different industries, performs medical analyzes, environmental factor analysis, etc. There are economic and production branches in which scientific research processes are carried out and in some cases they also use the results of chemical analysis.

Quality is a main topic in all fields and is therefore a problem that needs to be solved in the laboratories where various analyzes (chemical, physical, mechanical, etc.) are performed. Analyzes performed in chemical laboratories must provide accurate/real (qualitative, semi quantitative and quantitative) information on the elemental, functional and structural composition of any natural or synthetic compound. Under the implementation of the TQM concept, a theme of general interest in chemical laboratories is to assure and evaluate the quality of all activities that take place during the chemical analyzes. Maintaining quality control refers to the activities of supervising the performance of quality assessment processes in each of the stages of the chemical analysis process in order to eliminate and prevent nonconformities.

To address quality issues, quality management offers a number of methods, techniques and tools. Most of these tools are graphical methods because they clearly and explicitly express and analyze the problem. In the 1970s, the seven modern methods of quality management, also known as the New Seven, were defined: affinity diagram, relationship diagram, tree diagram, matrix diagram, diagrams of decisions (alternatives) factorial data. In many situations, we have non-numeric data and in such situations we can apply the modern tools of quality management that support the decisions for quality improvement.

Quality literature offers many articles and books that address the application of quality management tools. The tree chart has been used as a quality management tool in various areas for quality analysis and quality objectives. In Despotou [1], J ones and Arvanitis present the use of a tree diagram for designing a healthcare service. Another interesting work is [2], which presents a study of groundwater quality in which the decision tree method is used. Islam M.N. has published an article [3], in which he uses a tree diagram to determine the characteristics of a product according to customer requirements. The study presented in the paper [4] is based on the combined use of the fishbone chart and the tree diagram when managers want to improve the quality of proposals for science and technology programs. Menke, in his paper [5], presents the use of quality tools by companies that are concerned with improving the quality of $R \& D$ activities. A series of works, such as [6-12], present case studies on the analysis of non-quality problems using a classical fishbone diagram. In the paper [13], they present the defects of products that are obtained by forging and actions that can be taken to reduce these defects. In this paper, the author uses the tree diagram method. The tree diagram is also used in the studies presented in the papers [14,15]. The literature also includes a series of papers addressing the issue of continuous improvement of the quality of the processes carried out in different organizations based on the implementation of the TQM (Total Quality Management) concept, for example $[16,17]$.

Also, the issue of the quality of laboratory chemical analyzes is addressed in many scientific papers and specialized books in the field of chemistry. Olivares and Lopes in the paper [18] show the application of a practical instrument in analytical laboratories (including analytical chemistry laboratories). The tool used is the AQAC Analytical Quality Assurance Cycle and the laboratory tests performed are of high quality. The scientific article [19] provides guidance on the validation of laboratory analytical methods used in pharmaceutical analyzes for the purpose of quality assurance. EURACHEM and CITAC have developed a new guide [20], which provides guidance to laboratories on the application of best practices for analytical operations. The Guide addresses extensively quality issues: Measurement uncertainty, Methods / procedures for calibrations and tests, Method validation, Quality control and proficiency testing, etc. After 2002, the quality of laboratoryanalyzes has been increasingly concerned with the companies that manage such activities. California Resources Agency has published a Quality Assurance Manual for the Bryte Chemical Laboratory, [21]. The manual provides details on assuring and evaluating the quality of laboratory analyzes (Analytical Methods, Routine Procedures Used to Assess Data Quality, Acceptable Quality Control Limits, Internal Quality Control Checks, etc.).

\footnotetext{
*email: lylyanaluca@yahoo.com
} 
The ultimate goal of a chemical laboratory is to produce quality, accurate, accurate, complete, representative and compatible data. In this paper the authors propose to solve a quality problem in a chemical laboratory. Thus, the authors study the determination of the tree diagram to improve the quality of the analyzes performed in the chemical laboratory.

Determine and classify the measures necessary to improve the quality of analyzes performed in the chemical laboratory

The study aims to identify measures/actions that ensure the elimination of sources of errors that cause non-quality problems in analyzes performed in a chemical laboratory. The study is based on a quality management tool - tree diagram.

Several steps were taken to determine the tree diagram:

a. The problem to be solved is defined. The problem was formulated starting from the question How can we improve the quality of analyzes made in a chemical laboratory? Thus the following theme/problem was proposed: Improving the quality of analyzes performed in the chemical laboratory;

b. A Brainstorming meeting was held with specialists with competence in the field of chemistry and several measures/actions were proposed to solve the proposed topic;

c. The proposed measures have been grouped into several specific objectives correlated with the general objective (the problem/theme chosen);

$d$. The tree diagram was drawn based on the specific methodology.

The measures/actions proposed at the brainstorming session were complemented by other data obtained as a result of the study by the authors of specific publications in the literature. Thus all the measures identified for solving the problem were grouped on 4 specific objectives and are the following:

Specific Objective 1. The human resource shall be consistent with the required requirements.

Measures/Actions/Solutions:

M.1.1. Appropriate training of the laboratory analyst,

M.1.2. Laboratory operator with skills and practical abilities,

M.1.3. Laboratory analyst with appropriate theoretical and practical knowledge,

Secondary measures:

M.1.3.1. Knowledge of statistical processing,

M.1.3.2. Knowledge of how to prepare different chemical solutions,

M.1.3.3. Knowledge of laboratory analysis and sampling,

M.1.3.4. The correct interpretation of the results of the analyzes performed,

M.1.3.5. The correct estimation of uncertainties,

M.1.4. An operator who strictly adheres to standards and procedures,

M.1.5. Personally capable of observing phenomena during laboratory analysis,

M.1.6. Personally familiar with quality and quality records,

M.1.7. Staff with good health, good visual acuity, welldeveloped sense of smell,

M.1.8. Staff with individual and team work skills,

M.1.9. Responsible attitude,

M.1.10. Staff with abilities to communicate efficiently,

M.1.11. Personnel familiar with occupational safety and accident prevention measures.
Specific Objective 2. Use of appropriate equipment, tools and materials.

Measures/Actions/Solutions:

M.2.1. Equipment for laboratory analysis in the appropriate state,

Secondary measures:

M.2.1.1. Metrological verification and appropriate calibration,

M.2.1.2. Equipment with service contracts,

M.2.1.3. Equipment with maintenance plan,

M.2.1.4. Equipment meeting the requirements of the standards,

M.2.2. Apparatus for sampling in perfect condition,

M.2.3. Laboratory equipment according to the requirements of the analysis methods,

M.2.4. Preventive maintenance of laboratory instruments,

M.2.5. Supply of reagents and material for appropriate analyzes,

Secondary measures:

M.2.5.1. The reagents and materials used have a quality certificate from the manufacturer,

M.2.5.2. Reagents have prolonged stability,

M.2.5.3. Reagents and materials are used for shelf life,

M.2.5.4. Reagents are accurately weighed/measured.

Specific Objective 3. Applying appropriate methods and procedures.

Measures/Actions/Solutions:

M.3.1. The use of scientific, standardized and maximumaccuracy methods,

M.3.2. Choosing the right working methods (accuracy, detection limit, analytical interval, cost/effectiveness ratio)

M. 3.3. Validation of the method when using a method other than the reference method,

M. 3.4. Calibrating the method and verifying the calibration accuracy,

M. 3.5. Elaboration of protocols for chemical analyzes,

M. 3.6. Ensuring a proper time between sampling and testing,

M.3.7. Developing a sampling/measurement plan,

M.3.8. The correct choice of the sampling batch,

M.3.9. Appropriate sampling methods,

M.3.10. Keeping a sample of the samples taken and analyzed for further testing,

M.3.11. Using specific methodologies for research work. Specific Objective 4. Ensure a very good management. Measures/Actions/Solutions:

M.4.1. Top management is concerned with ensuring an appropriate environment,

Secondary measures:

M.4.1.1. The corresponding light,

M.4.1.2. Temperature and pressure corresponding to the requirements,

M.4.1.3. Working environment free of dust, noise, tremors, other noxious agents,

M.4.1.4. Appropriate environmental conditions.

M.4.2. Ensure effective and efficient management of human, material, and time resources,

M.4.3. Implement and maintain an integrated management system,

M.4.4. The periodic internal evaluation of the quality system

M.4.5. Top management to periodically analyze quality policy that must be in line with customer requirements,

M.4.6. Top management to ensure the necessary conditions for improving the quality, 
M.4.7. Policies for training and periodic training of laboratory staff,

M.4.8. Comparing laboratory performance with performance in other laboratories,

M.4.9. Regular visit of fairs for chemical laboratory equipment,

M.4.10. Periodic prospecting of reagents and equipment market,

M.4.11. Regular analysis of customer complaints (e.g. using the Pareto principle).

Determining the tree diagram to improve the quality of analyzes performed in the chemical laboratory

Measures have been identified and grouped into four specific objectives. According to the tree diagram methodology, in the case study presented it will be drawn with 4 levels. The first level of the diagram is represented by the proposed problem: Improving the quality of analyzes performed in the chemical laboratory. The second level of the tree diagram is represented by the four specific objectives: 1) The human resource to be consistent with the required requirements; 2) The use of adequate equipment, tools and materials; 3) Applying appropriate methods and procedures; 4) Ensuring a very good management.

Level III is formed with all measures/actions proposed for each specific objective. Some proposed measures have become secondary objectives and for these, secondary measures have been identified that form the IV level of the diagram.
The diagram is shown in figure 1 . For reasons of limited space, the proposed measures are given in the diagram only by the assigned code (e.g. M.1.1, M.1.2., ...) and also the secondary measures (M.1.3.1., M.1.3.2 ...). The diagram can be drawn on a large scale where all the proposed measures are written and the plan can be displayed in the chemical laboratory. In this case the diagram creates a complete visual image of the laboratory staff with the measures or actions to be taken to achieve the general objective: Improving the quality of analyzes performed in the chemical laboratory.

\section{Conclusions}

Quality management tools are a solution for analyzing and evaluating quality issues for products or services in many areas of activity. The study presented in the synthesis in this paper shows that it is possible to use the tree diagram and to solve a quality problem in a chemical laboratory. The study led to the identification of actions/measures that improve the quality of analyzes performed in the chemical laboratory. Several measures have been identified that have been grouped into four appropriate categories for four specific objectives: 1. Human resource to be appropriate to the required requirements; 2 . Use of appropriate equipment, tools and materials; 3. Application of methods and procedures appropriate to work; 4. Ensure a very good management.

A relatively complex tree diagram with a number of 4 levels has emerged. The obtained diagram offers a good graphic representation of the tasks, tools, measures or

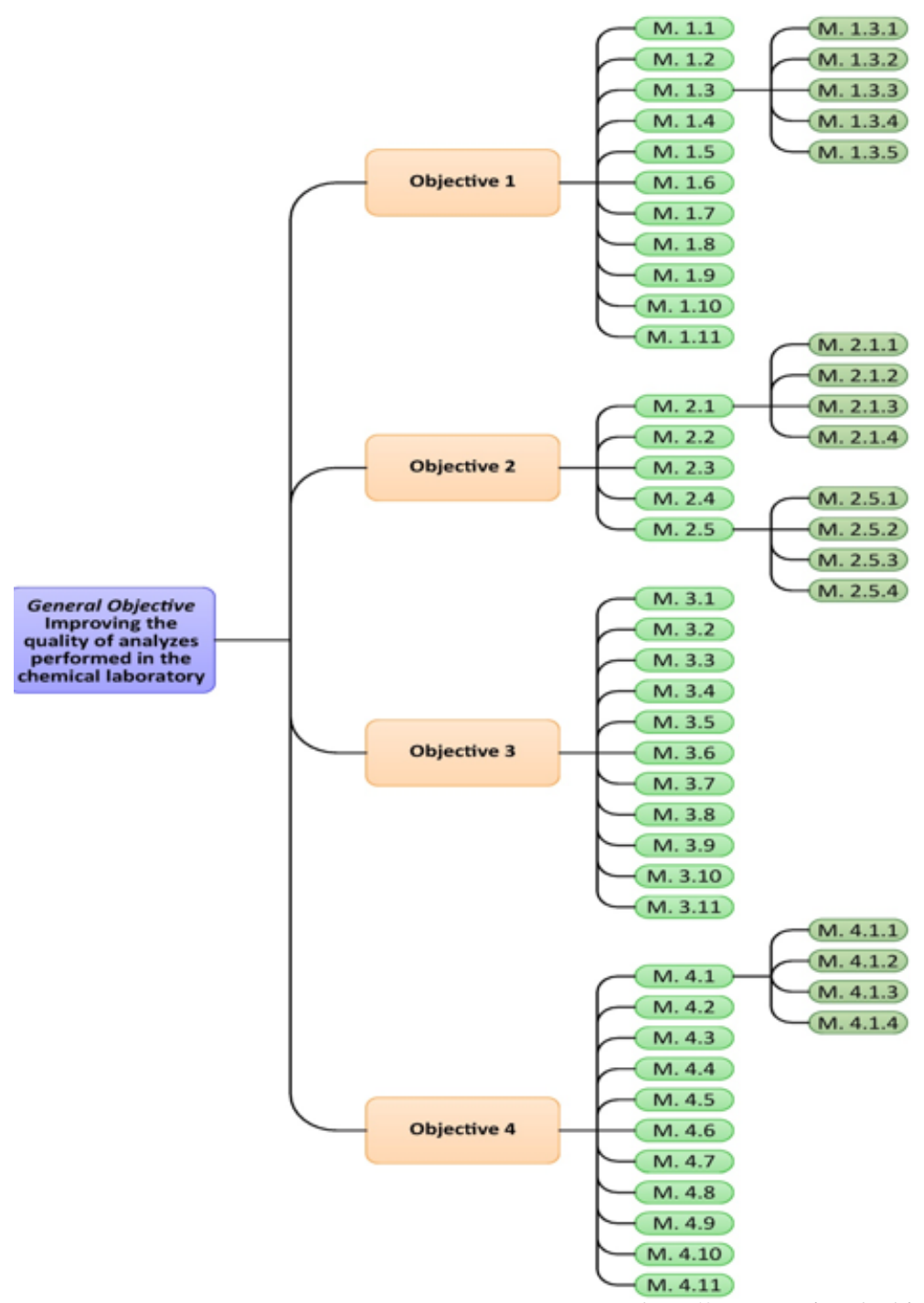

Fig. 1. Tree diagram for a chemical analysis laboratory 
actions to be taken in order to achieve the proposed general objective (the proposed problem) - Achieving high quality analyzes in a chemical laboratory. The diagram clearly and explicitly expresses the analysis and solving the proposed problem. The tree chart presented in the paper can help to achieve the end goal of a laboratory, to produce accurate, complete, representative, and compatible data. The tree chart proposed by the authors of the paper is a useful tool that helps the organization's management and staff in a chemical laboratory to improve the quality of laboratory analysis and thus increase customer satisfaction. The proposed measures can be further evaluated on the basis of efficiency and feasibility criteria and a priority can be given in their application.

\section{References}

1. DESPOTOU, G., J ONES, R.W., ARVANITIS, T.N., Using Event Trees to Inform Quantitative Analysis of Healthcare Services. Unifying the applications and foundations of biomedical and health informatics. Book Series: Studies in Health Technology and Informatics. Volume: 226, 2016, Pages: 119-122.

2. SAGHEBIAN, S.M., SATTARI, M.T., MIRABBASI, R., PAL, M., Ground water quality classification by decision tree method in Ardebil region, Iran. Arabian Journal of Geosciences. Volume:7 Issue: 11, 2014, Pages: 4767-4777.

3. ISLAM, M.N., A methodology for extracting dimensional requirements for a product from customer needs. International J ournal of Advanced Manufacturing Technology. Volume: 23 Issue: 7 8, 2004, Pages: 489-494.

4. LI, S.S., LEE, L.C., Using fishbone analysis to improve the quality of proposals for science and technology programs. Research Evaluation. Volume: 20, Issue: 4, 2011, Pages: 275-282.

5. MENKE, M.M., Improving research-and-development decisions and execution. Research-Technology Management. Volume: 37 Issue: 5, 1994, Pages: 25-32.

6. CIRTINA, L. M., CIRTINA, D., LUCA, L., Quality Management in Projects - Quality Planning, Applied Mechanics and Materials, Vol. 657, Trans Tech Publications, Switzerland, 2014, Pages: 891-895.

7. LUCA, L., CIRTINA, L. M., STANCIOIU, A., Study on Identification and Classification of Causes which Generate Welds Defects. Applied Mechanics and Materials, Volume: 657, Trans Tech Publications, Switzerland, 2014, Pages: 256-260.

8. LUCA, L., FILIP, C. P., On the assessment of the public order services quality by using classic instruments of quality management. 17th International Conference the Knowledge-Based Organization, Conference Proceedings 1: Management and Military Sciences, Book Series: Knowledge Based Organization International Conference, 2011, Pages: 691-696.
9. LUCA, L., A new model of Ishikawa diagram for quality assessment. Innovative Manufacturing Engineering \& Energy International Conference- IManEE 2016, Greece, Abstracts, ISBN 978-960-88944-9-5. IOP Conf. Series: Materials Science and Engineering 161 (2016) 012099 doi:10.1088/1757-899X/161/1/012099

10. LUCA, L., A study on quality analysis measuring process. Fiability \& durability, No 2, 2016, Pages: 68-72

11. LUCA, L., PASARE, M., STANCIOIU, A., Study to determine a new model of the Ishikawa diagram for quality improvement. Fiability \& durability, No 1, 2017, Pages: 249-254

12. LUCA, L., PASARE, M., Study on a new classification of causes which generate defects of injection molding products, Mat. Plast. 56, no. 1, 2019, p. 174-178

13. PASARE, M., On the mitigation actions of defects for plastical deformed parts. Fiability \& Durability/ Fiabilitate si Durabilitate, No. 2, 2016, Pages: 114-117

14. GHIMISI, S., NICULA, D., Actions to increase the reliability of chain transmissions, $22^{\text {nd }}$ International Conference on Innovative Manufacturing Engineering and Energy -IManE\&E 2018, Issue MATEC Web Conf., Volume 178, article number 06002, 2018, DOI:

15.GHIMISI, S., Measures for correct design, manufacturing and exploitation of gear transmissions to increase the reliability, Scientific Bulletin of Naval Academy, vol. 21, 2018, Constanta, Pages: 103-108

16. TODORU, A.V., Sustainable development of organizations through Total Quality Management, Procedia Social and Behovioral Sciences, Publish by Elsevier, Ltd, vol 62, 2012, Pages: 927-931

17. TODORU, A.V., The need of Total Quality Management in higher education, Procedia Social and Behovioral Sciences, Publish by Elsevier, Ltd, vol.83, 2013, Pages: 1105-1110

18. OLIVARES, I.R.B., LOPES, F.A., Essential steps to providing reliable results using the Analytical Quality Assurance Cycle, Trend in Analytical Chemistry, Vo. 35, 2012, Pages:109-121.

19. PANCHUMARTHY RAVISANKAR, CH. NAGA NAVYA, PRAVALLIKA, D., NAVYA SRI, D., A Review on Step-by-Step Analytical Method Validation, IOSR J ournal Of Pharmacy (e)-ISSN: 2250-3013, (p)-ISSN: 2319-4219 www.iosrphr.org Volume 5, Issue 10, 2015, Pages: 07-19

20. BARWICK, V. (Ed), Eurachem/CITAC Guide: Guide to Quality in Analytical Chemistry: An Aid to Accreditation (3rd ed. 2016). ISBN 9780-948926-32-7. Available from www.eurachem.org.

21. *** The Resources Agency, Bryte Chemical Laboratory Quality Assurance Manual, California, 2006. Available http://wwwdpla. water. ca.gov/supply/sampling/qa/p ublicat/Bryte.pdf

22.*** SR EN ISO/IEC 17025:2018, Cerine generale pentru competena laboratoarelor de încercãri 'i etalonãri.

23. *** SR EN ISO 9001:2015, Sisteme de managemental calitãii. Cerine. 24. *** SR EN ISO 9004:2010, Conducerea unei organizatii catre un succes durabil. $\mathrm{O}$ abordare bazata pe managementul calitatii

Manuscript received: 16.12 .2018 